\title{
Quantum Speedup for Index Modulation
}

\author{
Naoki Ishikawa, Member, IEEE.
}

\begin{abstract}
This letter presents a quantum-assisted index modulation for next-generation IoT wireless networks. The NP-hard index selection problem is first formulated by a quadratic unconstrained binary optimization problem consisting of constraints of feasible solutions. To minimize the number of qubits required for a quantum circuit, this formulation is then simplified by a dictionary-based approach that partially exploits a classical computer. It is observed that the Grover adaptive search can provide the quantum speedup for the index selection problem. This promising result implies that a future fault-tolerant quantum computer may be useful for solving optimization problems encountered in wireless communications.
\end{abstract}

Index Terms-Optimization, quantum computing, quantum speedup, MIMO, OFDM, index modulation, IoT.

\section{INTRODUCTION}

$\mathbf{I}$ NDEX modulation (IM) represents information by a combination of indices. Since Mesleh et al. proposed IM for multiple-input multiple-output (MIMO) scenarios [1], a tremendous number of papers have been published trying to break the trade-off between performance and computational complexity, especially in the field of wireless communications. The sparse structure of IM helps to reduce the number of multiplications. In particular, it has been demonstrated that significant gains can be achieved at low transmission rates, and it is expected to play an important role in IoT wireless networks [2]. Although this fact is not well-known, the first pioneering concept was established by Slepian in 1965 [3]. Beyond the communications field, applications similar to IM have been proposed in the fields of data storage and information hiding [4].

IM requires combinatorial optimization to determine which indices should be used. Specifically, any $K$ number of elements are selected from among $M$ elements such as transmit antennas or subcarriers. The corresponding number of patterns is $\left(\begin{array}{c}M \\ K\end{array}\right)$, which must be rounded down to a power of two, $Q$, to convey binary information. This optimization problem is NP-hard, and in this letter we refer to it as the index selection problem, which leads to a combinatorial explosion. Initially, the most common method was to use the first $Q$ rows of the combinatorial matrix [5]. The challenge with this method is that the selection probability of each element is unbalanced. To mitigate this unbalanced selection, Wen et al. proposed an efficient solution based on the greedy algorithm [6]. Although the computational complexity of [6] is negligible, the achievable minimum Hamming distance is two in any case, which limits its performance. In [7], the index selection problem is formulated as an integer linear programming problem. However, it cannot be solved on a classical computer depending on the size of the search space.

N. Ishikawa is with the Faculty of Engineering, Yokohama National University, 240-8501 Kanagawa, Japan (e-mail: ishikawa-naoki-fr@ynu.ac.jp).
Feynman invented computation by a reversible machine composed of unitary gates in 1986 [8]. In [8], he pointed out that the minimum energy required by a reversible machine is independent of the complexity of the calculation. Thus, a reversible quantum computer can be characterized by its power-saving performance. Quantum computers have promising advantages over classical computers in two major aspects: Shor's algorithm [9] for integer factoring in polynomial time and Grover search [10] that can provide the quantum speedup.

Grover search [10] can find a specific element from a database of unsorted $N$ elements with query complexity $O(\sqrt{N})$. This surprising property is called quadratic speedup, or quantum speedup. Similarly, in the case of the minimum value search, the query complexity is of the order of $O(\sqrt{N})$ [11]. The Grover search can be extended to solve minimization problems. This extended counterpart is termed Grover adaptive search (GAS) [12], and it can also provide the quantum speedup. The conventional GAS is unable to evaluate an objective function using a quantum oracle. In [13], Gilliam et al. solved this open issue by using a quantum dictionary to store key-value pairs in entangled quantum registers, which allows for the representation of a polynomial. It is shown in [13] that portfolio optimization, which is a quadratic unconstrained binary optimization (QUBO) problem, can be solved on a real quantum computer. Note that the quantum circuit for GAS requires qubits to encode an objective function, in addition to qubits for binary variables.

The variational quantum eigensolver [14] and the quantum approximate optimization algorithm (QAOA) [15] are wellknown methods for solving a QUBO problem with a reversible quantum computer. Another approach, quantum annealing [16, 17], may be useful in industrial applications [18] as it operates with low-latency, although its theoretical speedup has not been proven. Hence, this letter deals only with GAS that can provide the quantum speedup.

The authors of $[19,20]$ have led research in quantumassisted wireless communications. Specifically, Botsinis et al. used the Boyer-Brassard-Høyer-Tapp [21] and the DürrHøyer search algorithms [11] for performing maximum likelihood (ML) detection of discrete symbols on a quantum computer [22]. Besides, Matsumine et al. used QAOA for performing quasi-ML decoding of linear error-correcting codes. For more information on quantum optimization in wireless communications, a comprehensive survey can be found in [20].

Against this background, the NP-hard index selection problem is newly solved by GAS that can provide the quantum speedup. First, a straightforward constraint-based formulation is conceived for the problem. Next, a dictionary-based formulation is conceived to reduce the number of required qubits. This dictionary-based formulation defines the problem using good candidates that maximize the minimum Hamming 
distance, which is partially done by a classical computer. The number of qubits and the number of non-zero elements in the QUBO matrix are analyzed for both formulations. Finally, numerical analysis demonstrates a reduction in the average number of trials compared to the classic exhaustive search, confirming the quantum speedup as expected in theory.

\section{Conventional Index Modulation}

Index modulation (IM) conveys information by switching elements on and off. For example, part of transmit antennas are selected in the generalized spatial modulation [23], or part of subcarriers are selected in the subcarrier IM [24]. Any $K$ out of $M$ elements are selected, and the information is assigned to that selection pattern. This selection pattern can be defined by the combinatorial matrix $\mathbf{C}(M, K)$ in a recursive manner as follows [5]:

$$
\mathbf{C}(M, K)=\left[\begin{array}{cc}
\mathbf{1} & \mathbf{C}(M-1, K-1) \\
\mathbf{0} & \mathbf{C}(M-1, K)
\end{array}\right] \in \mathbb{B}\left(\begin{array}{c}
M \\
K
\end{array}\right) \times M
$$

Here, $\left(\begin{array}{l}M \\ K\end{array}\right)=M ! / K ! /(M-K) !$ denotes the binomial coefficient. In (1), 0 represents a zero vector of length $\left(\begin{array}{c}M-1 \\ K\end{array}\right)$, whereas 1 represents a one vector of length $\left(\begin{array}{l}M-1 \\ K-1\end{array}\right)$. If we have $(M, K)=(4,2)$, the combinatorial matrix is exemplified by

$$
\mathbf{C}(4,2)=\left[\begin{array}{llll}
1 & 1 & 0 & 0 \\
1 & 0 & 1 & 0 \\
1 & 0 & 0 & 1 \\
0 & 1 & 1 & 0 \\
0 & 1 & 0 & 1 \\
0 & 0 & 1 & 1
\end{array}\right] \in \mathbb{B}^{6 \times 4}
$$

Through the selected $K$ elements, $K$ complex-valued symbols $s_{1}, \cdots, s_{K} \in \mathbb{C}$ are transmitted, where the $L$-ary amplitude and phase-shift keying is used. The transmission rate is $R=B$ for generalized spatial modulation and $R=B / M$ for subcarrier IM, where the length of bits is

$$
B=\left\lfloor\log _{2}\left(\begin{array}{c}
M \\
K
\end{array}\right)\right\rfloor+K \log _{2}(L)[\mathrm{bit}]
$$

As an example, in the $(M, K)=(2,1)$ case, the combinatorial matrix is calculated as the identity matrix of size two, i.e., $\mathbf{C}(2,1)=\mathbf{I}_{2}$. Then, if we use $L=2$ constellation, the transmit vectors are given by $\left[\begin{array}{ll}+1 & 0\end{array}\right]^{\mathrm{T}},\left[\begin{array}{ll}-1 & 0\end{array}\right]^{\mathrm{T}},\left[\begin{array}{ll}0 & +1\end{array}\right]^{\mathrm{T}},\left[\begin{array}{ll}0 & -1\end{array}\right]^{\mathrm{T}} \in$ $\mathbb{C}^{M \times 1}$. For detailed construction methods, refer to the paper [7] that also provides an open-source implementation of IM.

\section{A. NP-Hard Index Selection Problem}

One of the significant problems of IM is the index selection problem. As given in (3), IM requires the binomial coefficient $\left(\begin{array}{l}M \\ K\end{array}\right)$ to be rounded down to a power of two, $Q$, to convey binary information. Specific $Q$ rows of the combinatorial matrix $\mathbf{C}(M, K)$ are used in the IM encoding. Note that $Q$ has to be within the region $2 \leq Q \leq Q_{\max }=2^{\left\lfloor\log _{2}\left(\begin{array}{c}M \\ K\end{array}\right)\right\rfloor}$. Since $\mathbf{C}(M, K)$ has $\left(\begin{array}{l}M \\ K\end{array}\right)$ rows, a critical problem arises: it is not obvious how $Q$ rows should be selected from $\mathbf{C}(M, K)$. The

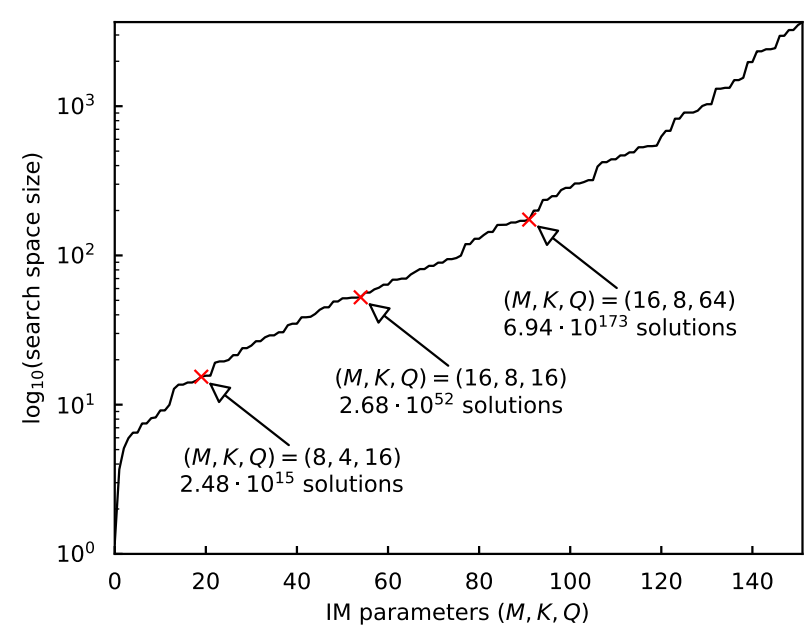

Fig. 1. The combinatorial explosion of the index selection problem, where the number of elements was increased from $M=2$ to 16 , the number of selected elements was varied from $K=2$ to $M-1$, and the number of selected rows was varied from $Q=2$ to $Q_{\max }$.

search space size of the index selection problem is quantified by

$$
S(M, K, Q)=\left(\begin{array}{c}
M \\
K \\
Q
\end{array}\right),
$$

which becomes large when $K$ is close to half of $M$ and when $Q$ is close to half of $Q_{\max }$.

More specifically, Fig. 1 exemplifies the search space size of the index selection problem. The IM parameters $(M, K, Q)$ were $2 \leq M \leq 16,2 \leq K \leq M-2$, and $2 \leq Q \leq Q_{\max }$, respectively. ${ }^{1}$ Additionally, only the cases where $Q \cdot K / M$ is an integer were considered, which will be described in Section III. As shown in Fig. 1, the search space becomes very large when we have $M \geq 8$. Specifically, in the $(M, K, Q)=(8,4,16)$ case, the search space size is approximately calculated as 2.48 . $10^{15}$. In the $(M, K, Q)=(16,8,64)$ case, we have about $6.94 \cdot 10^{173}$ solutions. Both cases indicate the difficulty of the index selection problem.

\section{Proposed Mathematical Formulations}

In this letter, the index selection problem is formulated by a QUBO problem and is solved by the GAS [13] that can provide the quantum speedup. In general, a QUBO problem is known as an NP-hard problem [25]. In the following, two formulations are newly conceived for the index selection problem. The first is a straightforward approach that only relies on constraints of variables. The second is an improved counterpart that exploits the combinatorial matrix. Both are termed constraint-based and dictionary-based formulations, respectively. Unlike the conventional approach [7], the proposed formulations are designed so that variables are binary and the corresponding objective function is a polynomial of the second degree that only returns an integer. Since GAS is used to solve a QUBO problem, the number of required

\footnotetext{
${ }^{1}$ For IM parameters $K=1, M-1$, and $M$, the corresponding solutions are obvious.
} 
qubits inevitably increases if the objective function returns a real number.

\section{A. Constraint-Based Formulation}

In this constraint-based approach, we focus only on constraints of feasible solutions that will be obtained. The solutions are binary variables with $Q$ rows and $M$ columns, and have two constraints:

1) the sum of each row is $K$ and

2) the sum of each column is $J$ on average, where $J=\lfloor Q \cdot K / M\rfloor$ has to be an integer. The corresponding objective function can be designed by

$$
\begin{aligned}
E_{1}(\mathbf{x}) & =\sum_{q=1}^{Q}\left(\sum_{m=1}^{M}\left(x_{q M+m}\right)-K\right)^{2} \\
& +\sum_{m=1}^{M}\left(\sum_{q=1}^{Q}\left(x_{q M+m}\right)-J\right)^{2} \in \mathbb{Z},
\end{aligned}
$$

where we have $n_{1}=Q M$ binary variables $\mathbf{x}=$ $\left[x_{1} \cdots x_{Q M}\right]^{\mathrm{T}} \in \mathbb{B}^{Q M \times 1}$. The maximum of $E_{1}(\mathbf{x})$ is derived as

$$
\max \left(E_{1}(\mathbf{x})\right)=\left\{\begin{array}{ll}
Q(M-K)^{2}+M(Q-J)^{2} & \left(K<\frac{M}{2}\right) \\
Q K^{2}+M J^{2} & \left(K \geq \frac{M}{2}\right)
\end{array},\right.
$$

and the number of qubits required to encode $E_{1}(\mathbf{x})$ is

$$
m_{1}=\left\lceil\log _{2}\left(\max \left(E_{1}(\mathbf{x})\right)\right)\right\rceil+1 \text {. }
$$

Finally, this constraint-based formulation requires $n_{1}+m_{1}$ qubits for the GAS circuit. According to algebraic analysis, the number of required qubits increases as $n_{1}+m_{1}=O(Q M)$, and the number of non-zero elements in the QUBO matrix of (5) increases as $Q M(Q+M) / 2=O(Q M(Q+M))$, where $O(\cdot)$ is an asymptotic notation.

As an example, for the IM parameters $(M, K, Q)=$ $(4,2,4)$, we have $n_{1}=Q M=16$ and $\max \left(E_{1}(\mathbf{x})\right)=$ $Q K^{2}+M J^{2}=16+16=32$ that results in $m_{1}=6$. Hence, the GAS circuit requires $n_{1}+m_{1}=16+6=22$ qubits for the proposed constraint-based formulation. Since this example originally has a small search space, i.e., $S(4,2,4)=\left(\begin{array}{l}6 \\ 4\end{array}\right)=15$, it is necessary to consider a formulation with fewer qubits.

\section{B. Dictionary-Based Formulation}

It is possible to reduce the number of qubits for the GAS circuit by reducing the number of variables and also narrowing the value range of the objective function. To reduce the number of variables, we limit solutions of the index selection to good candidates rather than formulating it only with constraints. Specifically, in the same manner as [7], we construct a submatrix of the combinatorial matrix $\mathbf{C}(M, K)$ defined in (1). Based on this submatrix, we again formulate the index selection problem as a simplified QUBO problem.

First, we construct a submatrix of $\mathbf{C}(M, K)$ that is composed of good candidates in terms of the minimum Hamming distance. For simplicity, $\mathbf{C}(M, K)$ is denoted by $\mathbf{C}^{(2)}$ because its minimum Hamming distance between each pair of rows is $H=2$. Later, the $i$-th row of $\mathbf{C}^{(2)}$ is denoted by $\mathbf{c}_{i} \in \mathbb{B}^{M}$ for $1 \leq i \leq\left(\begin{array}{l}M \\ K\end{array}\right)$. Let $H$ be the threshold of the minimum Hamming distance, the submatrix $\mathbf{C}^{(H)}$ is defined by a set of rows that satisfy $\operatorname{Hamming}\left(\mathbf{c}_{i}, \mathbf{c}_{j}\right) \geq H$ for $1 \leq i<j \leq\left(\begin{array}{l}M \\ K\end{array}\right)$. Here, Hamming $(\cdot, \cdot)$ denotes the Hamming distance between two binary vectors. For example, in the $(M, K)=(4,2)$ case, $\mathbf{C}^{(4)}$ is a submatrix of $\mathbf{C}^{(2)}$ as follows:

$$
\mathbf{C}^{(4)}=\left[\begin{array}{llll}
1 & 1 & 0 & 0 \\
0 & 0 & 1 & 1
\end{array}\right]
$$

that obviously satisfies the minimum Hamming distance $H=$ 4. A specific construction algorithm for $\mathbf{C}^{(H)}$ can be found in [7, Algorithm 3]. Note that the complexity imposed by this algorithm is negligible as compared to the complexity $S(M, K, Q)$ of the index selection problem.

Second, we find the minimum $H^{\prime}$ that satisfies $Q^{\prime}=$ nrow $\left(\mathbf{C}^{\left(H^{\prime}\right)}\right) \geq Q$ to determine which of submatrices $\left\{\mathbf{C}^{(2)}, \mathbf{C}^{(4)}, \mathbf{C}^{(6)}, \cdots\right\}$ should be used. For example, for the IM parameters $(M, K, Q)=(4,2,2)$, we can find the threshold $H^{\prime}=4$ and use the best dictionary $\mathbf{C}^{\left(H^{\prime}=4\right)}$ that has $Q^{\prime}=2$ rows.

Finally, the novel objective function can be designed by

$$
E_{2}(\mathbf{x})=\sum_{m=1}^{M}\left(\sum_{r=1}^{Q^{\prime}}\left(c_{r, m} \cdot x_{r}\right)-J\right)^{2} \in \mathbb{Z},
$$

where we have $J=\lfloor Q \cdot K / M\rfloor$ and $n_{2}=Q^{\prime}$ binary variables $\mathbf{x}=\left[\begin{array}{lll}x_{1} & \cdots & x_{Q^{\prime}}\end{array}\right]^{\mathrm{T}} \in \mathbb{B}^{Q^{\prime} \times 1}$. In (9), $c_{r, m}$ denotes the element at the $r$-th row and $m$-th column of the selected dictionary $\mathbf{C}^{\left(H^{\prime}\right)}$. The maximum of $E_{2}(\mathbf{x})$ is derived as

$$
\max \left(E_{2}(\mathbf{x})\right)=\left\{\begin{array}{ll}
K^{2}\left(Q^{\prime}-Q\right)^{2} / M & \left(Q<\frac{Q^{\prime}}{2}\right) \\
K^{2} Q^{2} / M & \left(Q \geq \frac{Q^{\prime}}{2}\right)
\end{array},\right.
$$

and the number of qubits required to encode $E_{2}(\mathbf{x})$ is

$$
m_{2}=\left\lceil\log _{2}\left(\max \left(E_{2}(\mathbf{x})\right)\right)\right\rceil+1 \text {. }
$$

The dictionary-based formulation can be further simplified by limiting $x_{1}=1$. Then, the required qubits is reduced from $n_{2}=Q^{\prime}$ to $n_{2}=Q^{\prime}-1$. The addition of this constraint can be expressed only by rewriting the objective function. According to algebraic analysis, the number of required qubits increases as $n_{2}+m_{2}=Q^{\prime}+2 \log _{2}(K)+\log _{2}(Q)=O\left(Q^{\prime}+\log (K)+\right.$ $\log (Q))$, and the number of non-zero elements in the QUBO matrix of (9) increases as $Q^{\prime 2} / 2=O\left(Q^{\prime 2}\right)$.

Let us check a specific example. In the $(M, K, Q)=$ $(4,2,4)$ case, we can find the threshold for the minimum Hamming distance $H^{\prime}=2$ and use the dictionary $\mathbf{C}^{\left(H^{\prime}=2\right)}$ defined in (2). The corresponding objective function is

$$
\begin{aligned}
E_{2}(\mathbf{x}) & =\sum_{m=1}^{4}\left(\sum_{r=1}^{6}\left(c_{r, m} \cdot x_{r}\right)-2\right)^{2} \\
& =\left(x_{1}+x_{2}+x_{3}-2\right)^{2}+\left(x_{1}+x_{4}+x_{5}-2\right)^{2} \\
& +\left(x_{2}+x_{4}+x_{6}-2\right)^{2}+\left(x_{3}+x_{5}+x_{6}-2\right)^{2} .
\end{aligned}
$$


Then, the optimal solution would be $E_{2}\left(\left[\begin{array}{llllll}1 & 1 & 0 & 0 & 1 & 1\end{array}\right]^{\mathrm{T}}\right)=0$ that indicates it is better to use the first, second, fifth, and sixth rows of $\mathbf{C}^{(2)}$.

For the simplified case that has $n_{2}=Q^{\prime}-1=5$, the number of required qubits is calculated as $n_{2}+m_{2}=10$, which is about half of that of the constraint-based formulation. We can limit $x_{1}=1$ and rewrite the objective function for $\mathbf{x}^{\prime}=\left[\begin{array}{lllll}x_{2} & x_{3} & x_{4} & x_{5} & x_{6}\end{array}\right]^{\mathrm{T}} \in \mathbb{B}^{5 \times 1}$ as $^{2}$

$$
\begin{aligned}
E_{2}\left(\mathbf{x}^{\prime}\right)= & \left(x_{2}+x_{3}-1\right)^{2}+\left(x_{4}+x_{5}-1\right)^{2} \\
& +\left(x_{2}+x_{4}+x_{6}-2\right)^{2}+\left(x_{3}+x_{5}+x_{6}-2\right)^{2} \\
= & -4 x_{2}^{2}+2 x_{2} x_{3}+2 x_{2} x_{4}+2 x_{2} x_{6}-4 x_{3}^{2}+2 x_{3} x_{5} \\
& +2 x_{3} x_{6}-4 x_{4}^{2}+2 x_{4} x_{5}+2 x_{4} x_{6}-4 x_{5}^{2} \\
& +2 x_{5} x_{6}-6 x_{6}^{2}+10=\mathbf{x}^{\prime \mathrm{T}} \mathbf{M} \mathbf{x}^{\prime}+10,
\end{aligned}
$$

where we have the QUBO matrix of

$$
\mathbf{M}=\left[\begin{array}{ccccc}
-4 & 2 & 2 & 0 & 2 \\
0 & -4 & 0 & 2 & 2 \\
0 & 0 & -4 & 2 & 2 \\
0 & 0 & 0 & -4 & 2 \\
0 & 0 & 0 & 0 & -6
\end{array}\right]
$$

We obtain the optimal solution $E_{2}\left(\left[\begin{array}{llll}1 & 0 & 0 & 1\end{array}\right]^{\mathrm{T}}\right)=0$, that indicates the second, fifth, and sixth rows should be used, in addition to the first row of (2). The number of non-zero elements in the QUBO matrix $\mathbf{M}$ is 13 , which is much smaller than that of the constraint-based formulation, 64 .

\section{NUMERICAL ANALYSIS}

In this section, both constraint-based and dictionary-based formulations are compared in terms of the complexity of quantum circuit. Then, the optimization process of GAS is demonstrated by simulations only for the dictionary-based formulation, which achieves better performance than the constraintbased formulation. IBM Qiskit [26] and its noisy quantum circuit simulator backend were used in the simulations.

First, in Fig. 2, the proposed constraint-based and dictionary-based formulations were compared in terms of the number of required qubits and the number of non-zero elements in the QUBO matrix, M. The horizontal axis of Fig. 2 corresponds to the index of IM parameters that were exactly the same as those used in Fig. 1. As shown in Fig. 2(a), the dictionary-based formulation succeeded in reducing the number of required qubits compared to the constraintbased formulation without any exception. Specifically, the dictionary-based formulation reduced the required qubits by $70.1 \%$ on average. Fig. 2(b) shows the number of non-zero elements in the QUBO matrix. While the QUBO matrix for the constraint-based formulation is sparse, it becomes dense in the dictionary-based case. It was observed that the constraintbased formulation outperformed the dictionary-based case in $73.7 \%$ of the total IM parameters. It can be seen from Fig. 2 that the numbers of required qubits and non-zero elements were in close agreement with the analysis of the derived order. The order depends only on $Q$ and $M$ in the constraint-based

\footnotetext{
${ }^{2}$ Note that we have $x_{2}^{2}=x_{2}$ as each variable is binary, i.e., $0^{2}=0$ and $1^{2}=1$.
}

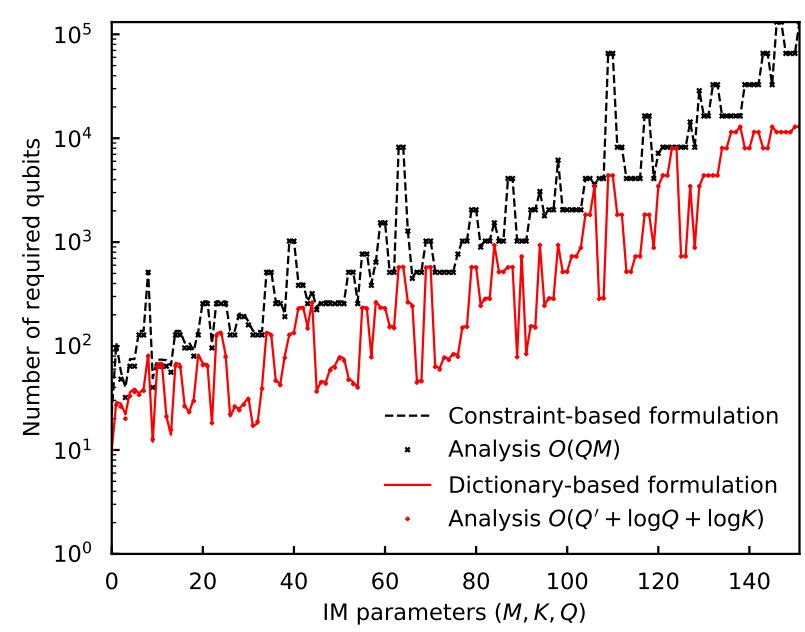

(a) The number of qubits.

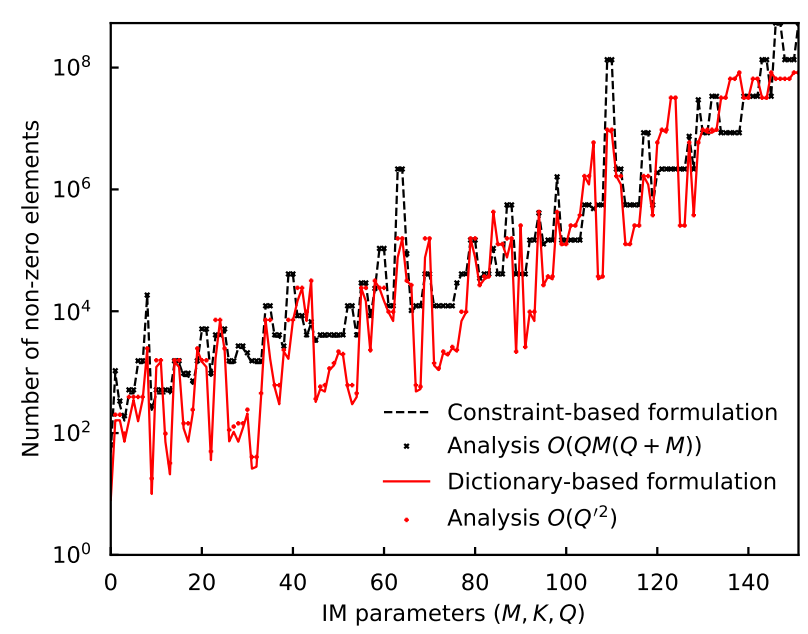

(b) The number of non-zero elements in $\mathbf{M}$.

Fig. 2. Comparisons between the constraint-based and dictionary-based formulations, where the IM parameters were the same as those used in Fig. 1.

case, while the order mainly depends on $Q^{\prime}$ in the dictionarybased case.

Second, as an example of the optimization process, Fig. 3 shows the output probabilities of each trial of GAS. In Fig. 3, the dictionary-based formulation having the IM parameters $(M, K, Q)=(4,2,4)$ was considered. A specific definition of the objective function $E_{2}\left(\mathbf{x}^{\prime}\right)$ is given in (13). The horizontal axis of Fig. 3 corresponds to $n_{2}=Q^{\prime}-1=5$ qubits, denoted by $\mathbf{x}^{\prime}=\left[\begin{array}{lllll}x_{2} & x_{3} & x_{4} & x_{5} & x_{6}\end{array}\right]$, where we have $2^{n_{2}}=32$ states. As shown in Fig. 3, two best solutions 13 and 19 were successfully marked by GAS. Specifically, the state ID $(13)_{10}=\left(\begin{array}{lllll}0 & 1 & 1 & 0 & 1\end{array}\right)_{2}$ corresponds to $\mathbf{x}^{\prime}=$ $\left[\begin{array}{lllll}0 & 1 & 1 & 0 & 1\end{array}\right]$, which indicates we should use $Q=4$ patterns: $\left[\begin{array}{llll}1 & 1 & 0 & 0\end{array}\right],\left[\begin{array}{llll}1 & 0 & 0 & 1\end{array}\right],\left[\begin{array}{llll}0 & 1 & 1 & 0\end{array}\right],\left[\begin{array}{llll}0 & 0 & 1 & 1\end{array}\right]$ extracted from (2). Additionally, the state ID $\left(\begin{array}{lll}19\end{array}\right)_{10}=\left(\begin{array}{lllll}1 & 0 & 0 & 1 & 1\end{array}\right)_{2}$ corresponds

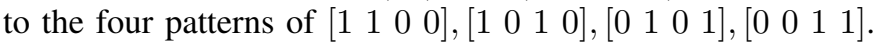
Both can achieve the best performance in terms of the bit error ratio and the average mutual information owing to $E_{2}\left(\left[\begin{array}{lllll}0 & 1 & 1 & 0 & 1\end{array}\right]\right)=E_{2}\left(\left[\begin{array}{lllll}1 & 0 & 0 & 1 & 1\end{array}\right]\right)=0$, which are optimal.

Finally, Fig. 4 shows the average of the objective function 


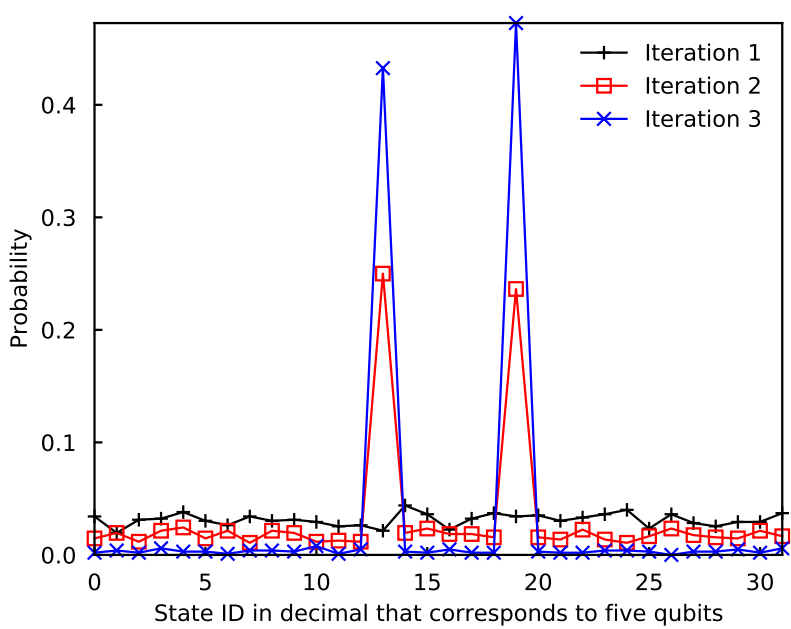

Fig. 3. Output probabilities of each GAS iteration for the dictionary-based formulation, where we had $(M, K, Q)=(4,2,4)$ and $2^{n_{2}}=32$ states.

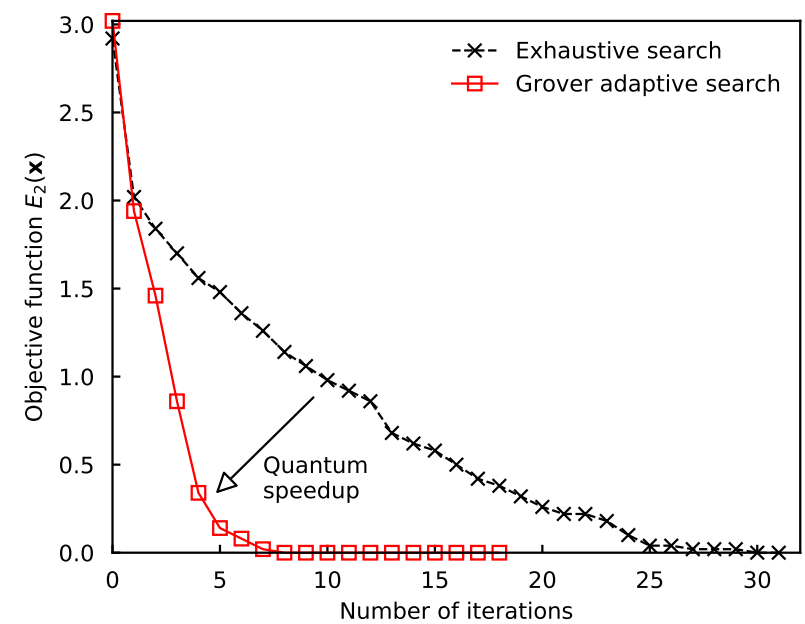

Fig. 4. The average of the objective function $E_{2}\left(\mathbf{x}^{\prime}\right)$ of (13) over each iteration.

$E_{2}\left(\mathbf{x}^{\prime}\right)$ corresponding to each iteration of the exhaustive search and GAS. As with Fig. 3, the IM parameters $(M, K, Q)=$ $(4,2,4)$ were considered. In this setup, the number of qubits was $n_{2}+m_{2}=5+\left\lceil\max \left(E_{2}\left(\mathbf{x}^{\prime}\right)\right)\right\rceil+1=5+4+1=10$. It was shown in Fig. 4 that the proposed formulation was able to provide the quantum speedup for the index selection problem.

\section{CONCLUSIONS}

In this letter, the NP-hard index selection problem, which determines the achievable performance of IM, was newly solved by the quantum-assisted GAS. The novel constraintand dictionary-based formulations were proposed for the problem. The numerical analysis demonstrated that the dictionarybased formulation was capable of reducing the required qubits and was able to provide the quantum speedup as expected in theory.

\section{ACKNOWLEDGEMENT}

IBM and Qiskit are trademarks of International Business Machines Corporation.

\section{REFERENCES}

[1] R. Y. Mesleh, H. Haas, S. Sinanovic, C. Ahn, and S. Yun, "Spatial modulation," IEEE Transactions on Vehicular Technology, vol. 57, no. 4 , pp. 2228-2241, 2008.

[2] N. Ishikawa, S. Sugiura, and L. Hanzo, "Subcarrier-index modulation aided OFDM - will it work?" IEEE Access, vol. 4, pp. 2580-2593, 2016.

[3] D. Slepian, "Permutation modulation," Proceedings of the IEEE, vol. 53, no. 3, pp. 228-236, 1965.

[4] N. Ishikawa, S. Sugiura, and L. Hanzo, "50 years of permutation, spatial and index modulation: From classic RF to visible light communications and data storage," IEEE Communications Surveys \& Tutorials, vol. 20, no. 3, pp. 1905-1938, 2018.

[5] P. K. Frenger and N. A. B. Svensson, "Parallel combinatory OFDM signaling," IEEE Transactions on Communications, vol. 47, no. 4, pp. 558-567, 1999.

[6] M. Wen, Y. Zhang, J. Li, E. Basar, and F. Chen, "Equiprobable subcarrier activation method for OFDM with index modulation," IEEE Communications Letters, vol. 20, no. 12, pp. 2386-2389, 2016.

[7] N. Ishikawa, "IMToolkit: An open-source index modulation toolkit for reproducible research based on massively parallel algorithms," IEEE Access, vol. 7, pp. 93 830-93 846, 2019.

[8] R. P. Feynman, "Quantum mechanical computers," Foundations of Physics, vol. 16, no. 6, pp. 507-531, 1986.

[9] P. Shor, "Algorithms for quantum computation: Discrete logarithms and factoring," in Annual Symposium on Foundations of Computer Science, Santa Fe, NM, USA, Nov. 20-22, 1994.

[10] L. K. Grover, "A fast quantum mechanical algorithm for database search," in ACM Symposium on Theory of Computing, Philadelphia, Pennsylvania, USA, May 22-24, 1996.

[11] C. Durr and P. Hoyer, "A quantum algorithm for finding the minimum," arXiv:quant-ph/9607014, 1999

[12] D. Bulger, W. P. Baritompa, and G. R. Wood, "Implementing pure adaptive search with Grover's quantum algorithm," Journal of Optimization Theory and Applications, vol. 116, no. 3, pp. 517-529, 2003.

[13] A. Gilliam, S. Woerner, and C. Gonciulea, "Grover adaptive search for constrained polynomial binary optimization," Quantum, vol. 5, 2021.

[14] A. Peruzzo, J. McClean, P. Shadbolt, M. H. Yung, X. Q. Zhou, P. J. Love, A. Aspuru-Guzik, and J. L. O'Brien, "A variational eigenvalue solver on a photonic quantum processor," Nature Communications, vol. 5, no. 1, 2014.

[15] E. Farhi, J. Goldstone, and S. Gutmann, "A quantum approximate optimization algorithm," arXiv:1411.4028, 2014.

[16] B. Apolloni, C. Carvalho, and D. de Falco, "Quantum stochastic optimization," Stochastic Processes and their Applications, vol. 33, no. 2, pp. 233-244, 1989.

[17] T. Kadowaki and H. Nishimori, "Quantum annealing in the transverse Ising model," Physical Review E, vol. 58, no. 5, pp. 5355-5363, 1998.

[18] T. Inagaki, Y. Haribara, K. Igarashi et al., "A coherent Ising machine for 2000-node optimization problems," Science, vol. 354, no. 6312, pp. 603-606, 2016.

[19] P. Botsinis, S. X. Ng, and L. Hanzo, "Quantum search algorithms, quantum wireless, and a low-complexity maximum likelihood iterative quantum multi-user detector design,” IEEE Access, vol. 1, pp. 94-122, 2013.

[20] P. Botsinis, D. Alanis, Z. Babar, H. V. Nguyen, D. Chandra, S. X. Ng, and L. Hanzo, "Quantum search algorithms for wireless communications," IEEE Communications Surveys \& Tutorials, vol. 21, no. 2, pp. 1209-1242, Secondquarter 2019.

[21] M. Boyer, G. Brassard, P. Hoyer, and A. Tapp, "Tight bounds on quantum searching," Fortschritte der Physik, vol. 46, no. 4-5, pp. 493505,1998

[22] P. Botsinis, D. Alanis, Z. Babar, S. X. Ng, and L. Hanzo, "Coherent versus non-coherent quantum-assisted solutions in wireless systems," IEEE Wireless Communications, vol. 24, no. 6, pp. 144-153, 2017.

[23] J. Jeganathan, A. Ghrayeb, and L. Szczecinski, "Generalized space shift keying modulation for MIMO channels," in IEEE International Symposium on Personal, Indoor and Mobile Radio Communications, Cannes, France, Sept. 15-18, 2008.

[24] E. Basar, U. Aygolu, E. Panayirci, and H. V. Poor, "Orthogonal frequency division multiplexing with index modulation," IEEE Transactions of Signal Processing, vol. 61, no. 22, pp. 5536-5549, 2013.

[25] A. Lucas, "Ising formulations of many NP problems," Frontiers in Physics, vol. 2, 2014

[26] G. Aleksandrowicz, T. Alexander, P. Barkoutsos et al., "Qiskit: An opensource framework for quantum computing," Zenodo, 2019. 